Die Abteilung Daten, Demographie und Qualität (DDQ) erstellt mit der Unterstützung der Arbeitsgruppe Qualität AGQ-FMH* Grundlagenpapiere zu verschiedenen Themen im Bereich Qualität, die in der Schweizerischen Ärztezeitung veröffentlicht werden. Die FMH nimmt auf der Basis der erarbeiteten Grundlagen Stellung zum Thema und gelangt über das Papier «Die Meinung der $\mathrm{FMH}$ » mit ihrer Position an die Öffentlichkeit. Nachfolgend werden das Grundlagenpapier sowie die Meinung der FMH zum Thema «Ärztliches Wohlbefinden und Behandlungsqualität» präsentiert.

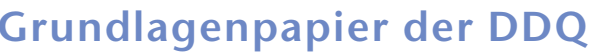

\section{Ärztliches Wohlbefinden beeinflusst die Behandlungsqualität}

\author{
Stefanie Hostettler, \\ Martina Hersperger, \\ Daniel Herren
}

\footnotetext{
* Mitglieder der AGQ-FMH (alphabetische Reihenfolge): Hélène Beutler, Karl Hampl, Catherine Heim, Daniel Herren, Martina Hersperger, Stefanie Hostettler,

Esther Kraft, Monika Loy, Francesca Mainieri, Varja Nikolic, Hans-Anton Vogel, Jürg von Below, Adrian Wirthner.

** Zur besseren Lesbarkeit wird nur die männliche Form verwendet; Frauen sind immer mitgemeint.
}

Korrespondenz:

FMH, Abteilung DDQ

Elfenstrasse 18

CH-3000 Bern 15

Tel. 0313591111

Fax 0313591112

ddq[at]fmh.ch

\begin{abstract}
Zusammenfassung
Obwohl die Arbeitsbelastung der Ärzte** im Allgemeinen hoch ist, sind sie mehrheitlich mit ihrer Berufssituation zufrieden. Bei einigen von ihnen führt die hohe Arbeitsbelastung aber zu einem chronischen Erschöpfungszustand und Burn-out bzw. reduziertem Wohlbefinden. Letzteres kann sich negativ auf die ärztliche Behandlungsqualität auswirken und die Patientensicherheit gefährden. Der vorliegende Artikel thematisiert die Einflussfaktoren und Ursachen von reduziertem Wohlbefinden der Ärzte sowie dessen Auswirkungen auf die Behandlungsqualität. Basierend auf den erarbeiteten Grundlagen ist es möglich, gezielte Präventionsmassnahmen zu ergreifen, so dass auch in Zukunft gesunde Ärzte für das Wohlergehen der Bevölkerung sorgen.
\end{abstract}

\section{Einleitung}

Die medizinische Versorgung in der Schweiz gehört zu den besten der Welt. Dies ist auch der qualitativ hochstehenden und engagierten Arbeit der Ärzteschaft zu verdanken. Es ist unbestritten, dass Ärzte signifikant zum Wohlergehen und zur Gesundheit der Bevölkerung beitragen und somit einen verantwortungsvollen Beruf ausüben. Viele Ärzte wählen ihren Beruf aus Überzeugung, zeichnen sich durch eine ausgeprägte Berufsidentifikation aus und leisten in der Regel einen überdurchschnittlichen Einsatz. Generell nehmen Ärzte die hohe Arbeitsbelastung in Kauf, weil der Arztberuf unter anderem ein interessantes Arbeitsumfeld, finanzielle Sicherheit sowie die Möglichkeit bietet, Menschen zu helfen. Zunehmend sind Ärzte jedoch mit hohen Patientenerwartungen und steigendem administrativen Aufwand konfrontiert. Lange Arbeitszeiten sowie Zeitdruck und -mangel gehören ebenfalls zum ärztlichen Berufsalltag. Trotzdem gelingt es der Mehrheit der
Ärzte, ihre Begeisterung und Energie für ihren Beruf ein Leben lang aufrechtzuerhalten: Sie würden sich ohne weiteres wieder für diesen Beruf entscheiden. Andererseits gibt es chronisch gestresste Ärzte, die durch die hohe Arbeitsbelastung Gefahr laufen, die Grenze zur Überlastung zu überschreiten [1, 2]. Längerfristig kann dies zu Energieverlust, Gereiztheit und Erschöpfung führen. Die Schwelle zur Überforderung ist individuell und wird grösstenteils auch durch persönliche Faktoren beeinflusst, wie etwa hohes Verantwortungsbewusstsein, Perfektionismus oder Wunsch nach Anerkennung [3].

Übermüdete und erschöpfte Ärzte neigen vermehrt dazu, Fehler zu begehen. Nicht unbeträchtlich ist, dass Folgen von medizinischen Fehlern die achthäufigste Todesursache sind: In den USA gehen jährlich zwischen 44000 bis 98000 vermeidbare Todesfälle darauf zurück $[4,5]$. Wahrscheinlich gibt es eine hohe Dunkelziffer an Fehlerereignissen, die aus Angst vor strafrechtlichen Folgen und Ansehensverlust verschwiegen werden $[1,6]$. Um die Öffentlichkeit vor Schäden zu schützen und eine qualitativ hochwertige Versorgung zu fördern, ist es wichtig, Fehlerursachen zu identifizieren und entsprechende Massnahmen zu ergreifen. In diesem Zusammenhang gehen wir hier der Frage nach, inwiefern das ärztliche Wohlbefinden die Behandlungsqualität oder Fehleranfälligkeit beeinflusst - ein bislang wenig beachteter Aspekt.

\section{Gesundheitszustand, Substanzmissbrauch und Suizid bei Ärzten}

Die körperliche Gesundheit der Ärzte unterscheidet sich im Allgemeinen nicht von derjenigen der Gesamtbevölkerung. Viele Studien zeigen auf, dass Ärzte risikoanfälliger für psychische Erkrankungen sind und unter ihnen Burn-out weit verbreitet ist [79]. Anzeichen und Auswirkungen von Burn-out sind unter anderem chronische Müdigkeit, Erschöpfung, Unkonzentriertheit, depressive Verstimmung und 


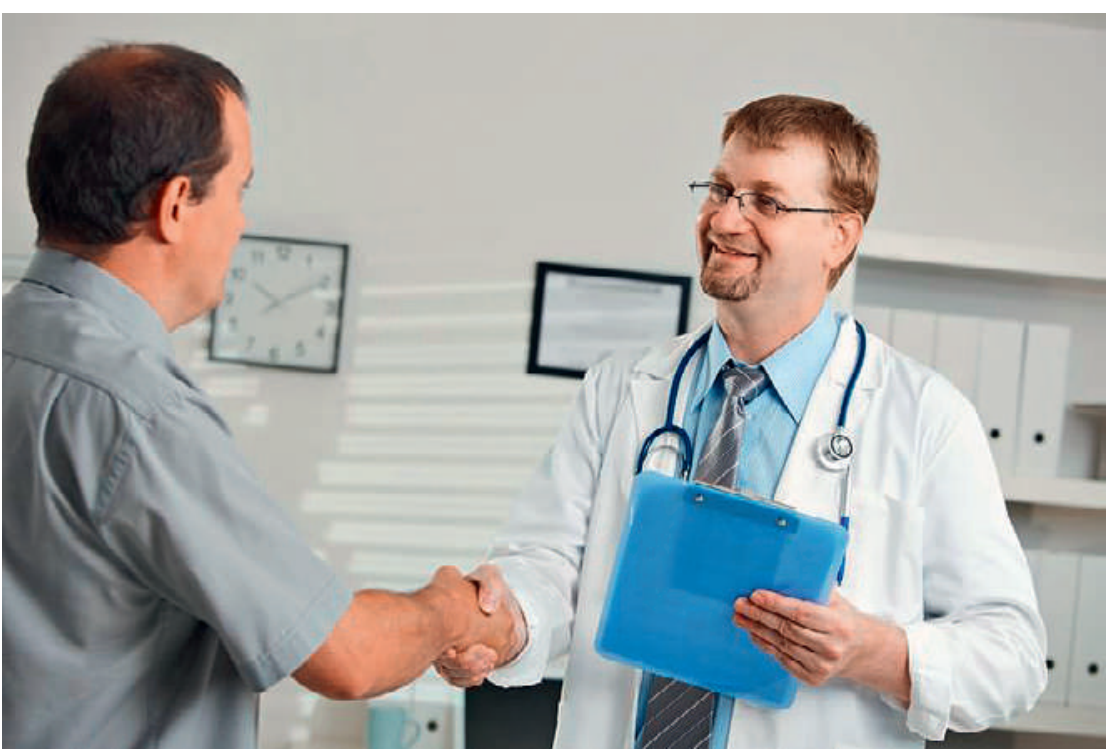

Ärzte nehmen eine hohe Arbeitsbelastung in Kauf, weil der Beruf ein interessantes Arbeitsumfeld bietet, finanzielle Sicherheit sowie die Möglichkeit, Menschen zu helfen.

innere Anspannung. Häufige Begleiterscheinungen bereits im Anfangsstadium der Erkrankung [10] sind Ängste, Fehler zu begehen, etwas zu übersehen oder sich vor Kollegen zu blamieren. Zu den vielschichtigen Ursachen von Burn-out gehören unter anderem Überforderung, chronisch hohe Stressexposition, Unsicherheit, Fehleranhäufung, hohes Arbeitspensum, Schlafentzug und emotionale Belastung [7]. Im Gegensatz zu ihren männlichen Kollegen sind Ärztinnen oft stärker herausgefordert, das Berufs- und Familienleben zu vereinbaren, was ihre Gesundheit beeinträchtigen kann $[2,11]$. scheinen auch Ärzte mit instabiler Kindheit im Erwachsenenalter anfälliger für Alkohol-, Drogen- oder Medikamentenmissbrauch zu sein [2].

Suizid ist der häufigste Grund für vorzeitigen Tod unter Ärzten und die zweithäufigste Todesursache bei Medizinstudenten [10]. Ärzte sind somit 2,45mal mehr suizidgefährdet als die Gesamtbevölkerung [15]. Auffallend ist, dass Suizide unter Landärzten und Psychiatern häufiger vorkommen als bei Stadtärzten und Ärzten der restlichen Fachrichtungen. Risikofaktoren für Suizide wie psychische Erkrankungen und Substanzmissbrauch können unter Ärzten verbreitet vorkommen und bleiben nicht ohne Folgen für die ärztliche Arbeit [16-18]. Ärzte, denen es nicht gutgeht, arbeiten mit weniger Überzeugung und Zufriedenheit, sind weniger produktiv und gehen schlechter auf Patienten ein, was sich letztlich auch auf die Behandlungsqualität auswirkt $[2,18,19]$.

\section{Ärztliches Wohlbefinden und Behandlungsqualität}

Der Begriff der Behandlungsqualität umfasst eine Vielzahl von Aspekten und Merkmalen: Sie wird etwa an der Patientenzufriedenheit, an Fehlerereignissen oder an professionellem Verhalten gemessen.

Reduziertes ärztliches Wohlbefinden kann sowohl zu einer schlechteren Arzt-Patienten-Kommunikation als auch zu Stress und Zeitdruck führen. Dadurch werden nicht alle Behandlungsmöglichkeiten ausgeschöpft und wichtige Tests unterlassen mit einer suboptimalen Behandlungsqualität als Ergebnis [20-22]. Beispielsweise gaben $44 \%$ von 1311 chirurgisch tätigen Ärzten aus 489 Spitälern an, dass die Qualität der Patientenversorgung durch

\section{Hauptgründe für Stress: hohes Arbeitsvolumen, zu wenig Schlaf, Unsicherheit bei Diagnosen und die Anforderung, das Wissen auf dem aktuellen Stand zu halten.}

Medikamenten- und Drogenmissbrauch ist unter Ärzten im Vergleich zur Allgemeinbevölkerung höher, wobei insbesondere die leichte Erreichbarkeit und Selbstverschreibung bei Medikamenten ein Schlüsselfaktor ist [12]. Medikamente und Drogen werden dabei primär zur Schmerzerleichterung und als Aufputschmittel konsumiert, um längere Dienste zu überstehen, nicht um Rauschzustände zu erreichen [13]. Aus Angst vor beruflichen Konsequenzen und Imageverlust wird die Sucht oftmals nach aussen bagatellisiert und verleugnet. Zum Alkoholkonsum bestehen kontroverse Resultate: Einige Studien stellten einen erhöhten Alkoholkonsum unter Ärzten fest [13], in anderen wiederum ist dieser vergleichbar mit demjenigen von ähnlichen sozialen Schichten [14]. Analog zur Gesamtbevölkerung
Überarbeitung beeinträchtigt sei [23]. Nachweislich verursacht Burn-out eine höhere Fehleranfälligkeit, etwa beim Verschreiben von Medikamenten [2, 7, 24, 25].

Befragungen von Ärzten über mehrere Monate hinweg zu Lebensqualität, Burn-out, Depression und Fehlerereignissen zeigten, dass sich die Fehlerhäufigkeit umgekehrt zur Lebensqualität und proportional zu Burn-out und Depression verhält [4, 26, 27]. Diese Symptome erhöhen gemeinsam mit einer tieferen Lebensqualität gleichzeitig die Wahrscheinlichkeit, auch in Zukunft Fehler zu begehen [26]. Hinzu kommt, dass die auf diese Weise angeschlagene psychische Belastung (z. B. Selbstzweifel, Angst, Versagensängste) weitere Fehlerereignisse begünstigt [4] und so zu einem reziproken Zyklus wird [28]. 
Als Folge von hohem Arbeitspensum und Mehrfachbelastungen kämpfen fast alle Ärzte im Laufe ihrer Karriere mit Schlafmangel und Müdigkeit, die Fehlerereignisse, Aufmerksamkeitsdefizite und Leistungsverminderung nach sich ziehen [1, 21, 29-31]. Der Bewusstseinszustand und die Leistungsfähigkeit nach einer 24-Stunden-Schicht sind vergleichbar mit einem angetrunkenen Zustand; daneben nimmt die Gefahr zu, nach langen Schichten einen Verkehrsunfall $\mathrm{zu}$ verursachen. Erholung und ausreichend Schlaf sind somit wichtige Faktoren im Zusammenhang mit der Patientensicherheit und Behandlungsqualität [31-33].

Demgegenüber zeigen auch Studien, dass sich eine hohe Arbeitsbelastung nicht per se negativ auf die Patientensicherheit auswirkt: Nach der Reduktion der ärztlichen Arbeitszeitlimite sind die Komplikationsrate und Mortalität von operierten Patienten nicht gesunken $[34,35]$.

\section{Situation in der Schweiz}

Viele Studien zur ärztlichen Gesundheit und Behandlungsqualität stammen aus dem englischsprachigen Raum, wo sich die Arbeitsbedingungen nicht zwingend mit den hiesigen vergleichen lassen. 2005 wurde in der Schweiz eine gesetzliche Arbeitszeitbeschränkung für Assistenz- und Oberärzte eingeführt, die maximal 50 Stunden pro Woche für die Assistenzärzte beziehungsweise 46 Stunden pro Woche für Oberärzte vorschreibt. Jedoch ist davon auszugehen, dass diese maximalen Arbeitszeiten häufig überschritten und Überstunden nicht aufgeschrieben werden - Ärzte geraten durch die zeitliche Beschränkung womöglich noch stärker unter Stress und Zeitdruck.

Im Vorfeld zur Einführung der neuen Spitalfinanzierung wurden Schweizer Spitalärzte und frei praktizierende Ärzte zu ihrer Arbeitszufriedenheit befragt. Diese Selbsteinschätzung fiel trotz hohem Zeit- und Leistungsdruck mehrheitlich positiv aus. Dennoch gaben die Befragten an, neben Stress mindestens hie und da an Beziehungsproblemen (29\% Spitalärzteschaft, $28 \%$ praxisambulant tätige Ärzteschaft) und Burn-out (22\% Spitalärzteschaft, 25\% praxisambulant tätige Ärzteschaft) zu leiden [36]. Ausserdem scheint die zunehmende Bürokratisierung des Berufs die praktizierenden Ärzte zu belasten.

Eine Befragung zum Gesundheitszustand der Schweizer Hausärzte zeigte, dass ein Drittel der Grundversorger Anzeichen von Burn-out haben [37] und dass bei 44 Prozent der Befragten der berufliche Stress stark oder sehr stark ist [14]. Als Hauptgründe für den Stress wurden genannt: das hohe Arbeitsvolumen, zu wenig Schlaf, Unsicherheit bei Diagnosen und die Anforderung, das Wissen auf dem aktuellen Stand zu halten. Zwei Drittel der Ärzte sind auch der Ansicht, dass ihr Privatleben beeinträchtigt ist. Bedenklich ist der relativ hohe Medikamentenkonsum:
65\% der Hausärzte gaben an, in den letzten sieben Tagen Medikamente (34\% Schmerzmittel, 13\% Antihypertensiva, 9\% Benzodiazepine, 7\% Antidepressiva) eingenommen zu haben [14].

In einer Literaturübersicht zum Wohlbefinden der Ärzte in der Schweiz wurde deutlich, dass einerseits die abnehmende Wertschätzung des Arztberufs und andererseits die zunehmende Einschränkung der Autonomie den Stress verschlimmern und das Wohlbefinden beinträchtigen [13]. Die Autoren betonen, dass das Wohlbefinden der Ärzte in der Schweiz einen höheren Stellenwert haben sollte die ärztliche Versorgungsqualität hängt unter anderem davon ab.

Tatsächlich hat eine Schweizer Studie [38] zu den Auswirkungen des ärztlichen Wohlbefindens aufgezeigt, dass eine hohe Arbeitsbelastung und emotionale Erschöpfung unter Assistenz- und Oberärzten vermehrt zu Aversionen gegen Patienten führten. Dies ist insbesondere deswegen ungünstig, da der Arztberuf durch eine hohe Anzahl an Patientenkontakte gekennzeichnet ist und die Ärzte sich auch mit schwierigen Patientencharakteren auseinandersetzen müssen.

\section{Präventionsmassnahmen}

Ärzte nehmen sich selten als Patienten wahr und zögern, Hilfe in Anspruch zu nehmen - dies würde wohl als Zeichen von Schwäche und Inkompetenz gelten. Deshalb ist es umso wichtiger, Präventionsmassnahmen über verschiedene Quellen und arztnahe Organisationen zu unterstützen. Diese können Ärzte eher dazu anregen, einen gesunden Lebensstil $\mathrm{zu}$ führen, mehr Ruhepausen einzuschalten, falls nötig das Arbeitspensum zu reduzieren und das soziale Leben ausserhalb der Praxis oder des Spitals zu pflegen [19]. Es wäre zu begrüssen, wenn Ärzte besser die Zeichen der Erschöpfung wahrnehmen und mehr auf ihre eigenen Bedürfnisse achten.

Künftig sollten gezielte Massnahmen zur Sicherung der ärztlichen Qualität vermehrt gefördert werden, wie zum Beispiel Qualitätszirkel, Supervisionen oder Fallbesprechungen. Die Teilnahme an einem anonymen Fehlermeldesystem wie CIRS (Critical Incident Reporting System) fördert das Bewusstsein für Fehler und bietet die Gelegenheit, aus ihnen zu lernen. Das Projekt «Täter als Opfer» der Stiftung für Patientensicherheit führt Schulungen für Kaderärzte durch, um den konstruktiven Umgang mit Fehlern in Gesundheitsorganisationen zu verbessern.

In der Schweiz erhalten Ärzte in schwierigen Situationen Hilfe bei ReMed, einem Unterstützungsnetzwerk für Ärzte. Dieses hat zum Ziel, dank gesunden Ärzten einen Beitrag zur Qualität der medizinischen Versorgung zu leisten [39]. Seit 2008 konnten qualifizierte Ärzte Kollegen weiterhelfen, die überlastet sind, die an Depressionen leiden oder denen der Tod von Patienten zu schaffen macht. Das Netzwerk bietet für Ärzte in schwierigen Lebenssituationen 
Mentoring, Krisenintervention, Beratung, Coaching, Therapie und Assessments; die Unterstützungsmassnahmen werden an die Situation der Hilfesuchenden angepasst.

\section{Schlussfolgerung}

Obwohl viele verschiedene Faktoren das ärztliche Wohlbefinden bestimmen, beeinflusst dieses entscheidend die Behandlungsqualität. Gesunde und zufriedene Ärzte leisten mehr, haben weniger Absenzen und machen weniger Fehler - dies hat neben der erhöhten Patientensicherheit auch einen sozioökonomischen Nutzen. Gezielte Unterstützungsmassnahmen sollten in enger Zusammenarbeit mit Fachgesellschaften erarbeitet werden, um fachspezifische

\section{Eine hohe Arbeitsbelastung und emotionale Erschöpfung führen vermehrt zu Aversionen gegen Patienten.}

Belastungen besser zu berücksichtigen. Zudem sollten im Schweizer Politsystem die Voraussetzungen für Reformen geschaffen werden, um den bevorstehenden Herausforderungen des Gesundheitssystems wie Mangel an Fachkräften und Ärzten entgegenzuwirken.

Es ist davon auszugehen, dass sich die Arbeitsbelastung für Ärzte in Zukunft durch die mit Personaleinsparung und Sparmassnahmen gepaarte finanzielle und personelle Ressourcenknappheit erhöhen wird. Ausserdem führt die gesellschaftliche Entwicklung zu einer zunehmenden Überalterung und einem Anstieg von schwerkranken Patienten, was bestimmte Ärztegruppen emotional und zeitlich zusätzlich belasten kann.

Bislang haben nur wenige wissenschaftliche Studien den Zusammenhang zwischen dem ärztlichen Wohlbefinden und der Behandlungsqualität in der Schweiz untersucht. Für eine gezielte und nachhaltige Forschung auf diesem Gebiet ist ein Instrument notwendig, das diese beiden Aspekte erfasst und eine Bestandesaufnahme nach dem heutigen Reglement (z. B. Arbeitszeitbeschränkung) erlaubt.

Rund um das Thema ärztliches Wohlbefinden und Behandlungsqualität bleiben weiterhin zahlreiche Fragen offen. Insbesondere wäre es wichtig zu wissen, ob es zwischen dem ambulanten und stationären Sektor Unterschiede gibt, und die Fachrichtungen zu identifizieren, bei denen sich das ärztliche Wohlbefinden messbar auf die Behandlungsqualität auswirkt.

Mit der Erlangung neuer Erkenntnisse ist es möglich, gezielte Präventionsmassnahmen zu ergreifen, so dass auch in Zukunft gesunde Ärzte für das Wohlergehen der Bevölkerung sorgen.

\section{Literatur}

1 Wallace JE, Lemaire JB, Ghali WA. Physician wellness: a missing quality indicator. Lancet 2009;374:1714-21.

2 Spickard A, Gabbe SG, Christensen JF. Mid-career burnout in generalist and specialist physicians. JAMA 2002;288:447-50.

3 Schütz E. Burn-Out-Syndrom: Die drei grossen «C». Österreichisches Ärzteblatt. 2008;12:22-4.

4 Shanafelt TD, Balch CM, Bechamps G, Russell T, Dyrbye L, Satele D, et al. Burnout and medical errors among American surgeons. Ann Surg. 2010;251:995-1000.

5 Sexton JB, Thomas EJ, Helmreich RL. Error, stress, and teamwork in medicine and aviation: cross sectional surveys. BMJ 2000;320:745-9.

6 O'Neil E. Leading to well-being: the 5 key elements of healthy leadership. West J Med. 2001;174:34-6.

7 Shanafelt TD, Bradley KA, Wipf JE, Back AL. Burnout and self-reported patient care in an internal medicine residency program. Ann Intern Med. 2002;136:358-67.

8 Remen RN. Recapturing the soul of medicine: physicians need to reclaim meaning in their working lives. West J Med. 2001;174:4-5.

9 Deckard G, Meterko M, Field D. Physician burnout: an examination of personal, professional, and organizational relationships. Med Care. 1994;32:745-54.

10 Bergner TMH. Burnout bei Ärzten. Stuttgart: Schattauer Verlag; 2006.

11 Robinson GE. Stresses on women physicians: consequences and coping techniques. Depress Anxiety 2003;17:180-9.

12 Gundersen L. Physician burnout. Ann Intern Med. 2001;135:145-8.

13 Kursner D, Danuser B. Arbeitsmedizin: Die Arbeitsmedizin nimmt die psychische Gesundheit der Schweizer Ärztinnen und Ärzte unter die Lupe. Schweiz Ärztezeitung. 2007; 88(7):7-8.

14 Bovier P, Bouvier Gallacchi M, Goehring C, Künzi B. Wie gesund sind die Hausärzte in der Schweiz? Primary Care. 2005;5:222-8.

15 Stack S. Suicide risk among physicians: a multivariate analysis. Arch Suicide Res. 2004;8:287-92.

16. Dyrbye LN, Massie FS, Jr., Eacker A, Harper W, Power D, Durning SJ, et al. Relationship between burnout and professional conduct and attitudes among US medical students. JAMA 2010;304:1173-80.

17 Ramirez AJ, Graham J, Richards MA, Cull A, Gregory WM. Mental health of hospital consultants: the effects of stress and satisfaction at work. Lancet 1996;347:724-8.

18 Parker PA, Kulik JA. Burnout, self- and supervisor-rated job performance, and absenteeism among nurses. J Behav Med. 1995;18:581-99.

19 Haas JS, Cook EF, Puopolo AL, Burstin HR, Cleary PD, Brennan TA. Is the professional satisfaction of general internists associated with patient satisfaction? J Gen Intern Med. 2000;15:122-8.

20 West CP, Shanafelt TD, Kolars JC. Quality of life, burnout, educational debt, and medical knowledge among internal medicine residents. JAMA 2011;306:952-60. 
21 Firth-Cozens J, Greenhalgh J. Doctors' perceptions of the links between stress and lowered clinical care. Soc Sci Med. 1997;44:1017-22.

22 Levin S, Aronsky D, Hemphill R, Han J, Slagle J, France DJ. Shifting toward balance: measuring the distribution of workload among emergency physician teams. Ann Emerg Med. 2007;50:419-23.

23 Knesebeck O, Klein J, Grosse K, Blum K, Siegrist J. Psychosoziale Arbeitsbelastung bei chirurgisch tätigen Krankenhausärzten. Deutsches Ärzteblatt. 2010;107:248-53.

24 Fahrenkopf AM, Sectish TC, Barger LK, Sharek PJ, Lewin D, Chiang VW, et al. Rates of medication errors among depressed and burnt out residents: prospective cohort study. BMJ 2008;336:488-91.

25 Chopra SS, Sotile WM, Sotile MO. Physician burnout. JAMA. 2004;291:633.

26 West CP, Tan AD, Habermann TM, Sloan JA, Shanafelt TD. Association of resident fatigue and distress with perceived medical errors. JAMA. 2009;302:1294-300.

27 West CP, Huschka MM, Novotny PJ, Sloan JA, Kolars JC, Habermann TM, et al. Association of perceived medical errors with resident distress and empathy: a prospective longitudinal study. JAMA. 2006;296:1071-8.

28 Schwappach DL, Boluarte TA. The emotional impact of medical error involvement on physicians: a call for leadership and organisational accountability. Swiss Med Wkly. 2009;139:9-15.

29 Lockley SW, Cronin JW, Evans EE, Cade BE, Lee CJ, Landrigan CP, et al. Effect of reducing interns' weekly work hours on sleep and attentional failures. N Engl J Med. 2004;351:1829-37.
30 Barger LK, Ayas NT, Cade BE, Cronin JW, Rosner B, Speizer FE, et al. Impact of extended-duration shifts on medical errors, adverse events, and attentional failures. PLoS Med. 2006;3:e487.

31 Weinger MB, Ancoli-Israel S. Sleep deprivation and clinical performance. JAMA. 2002;287:955-7.

32 Gaba DM, Howard SK. Patient safety: fatigue among clinicians and the safety of patients. N Engl J Med. 2002;347:1249-55.

33 Gottlieb DJ, Parenti CM, Peterson CA, Lofgren RP. Effect of a change in house staff work schedule on resource utilization and patient care. Arch Intern Med. 1991;151:2065-70.

34 Kaderli R, Businger A, Oesch A, Stefenelli U, Laffer U. Morbidity in surgery: impact of the 50-hour work-week limitation in Switzerland. Swiss Med Wkly. 2012;142:0, doi: 10.4414/smw.2012.13506.

35 Poulose BK, Ray WA, Arbogast PG, Needleman J, Buerhaus PI, Griffin MR, et al. Resident work hour limits and patient safety. Ann Surg. 2005;241:847-56.

36 www.fmh.ch/tarife/begleitforschung.html. 2011.

37 Goehring C, Bouvier Gallacchi M, Kunzi B, Bovier P. Psychosocial and professional characteristics of burnout in Swiss primary care practitioners: a cross-sectional survey. Swiss Med Wkly. 2005;135:101-8.

38 Biaggi P, Peter S, Ulich E. Stressors, emotional exhaustion, and aversion to patients in residents and chief residents - what can be done? Swiss Med Wkly. 2003;133:339-46.

39 Peltenburg M, Hersperger M, Stucki I, Tschan W. Projekt ReMed: Wer wendet sich an das Unterstützungsnetzwerk? Schweiz Ärztezeitung. 2008;89(50):2153-5. 